\title{
CONCURRENT VALIDITY AND RELIABILITY OF THE LOAD-VELOCITY RELATIONSHIP TO PREDICT THE ONE-REPETITION MAXIMUM DURING THREE WEIGHTLIFTING DERIVATIVES
}

\author{
Ricardo Berton', Marcos Soriano², Demostenys David da Silva1, Marcel Lopes dos Santos ${ }^{3}$, \\ Gustavo Teixeira ${ }^{1}$, Fernando Reiser ${ }^{4}$, and Valmor Tricoli ${ }^{1}$ \\ ${ }^{1}$ School of Physical Education and Sport, University of São Paulo, São Paulo, Brazil \\ ${ }^{2}$ Strength Training \& Neuromuscular Performance (STreNgthP) Research Group, \\ Camilo José Cela University, Madrid, Spain \\ ${ }^{3}$ School of Kinesiology, Applied Health, and Recreation, \\ Oklahoma State University, Stillwater, USA \\ ${ }^{4}$ School of Arts, Sciences and Humanities, University of São Paulo, São Paulo, Brazil
}

Original scientific paper

DOI $10.26582 / \mathrm{k} .53 .2 .4$

\begin{abstract}
:
The study investigated the concurrent validity and reliability of the load-velocity relationship to predict the one-repetition maximum (1RM) of the power clean from the knee (PCK), high pull from the knee (HPK), and mid-thigh clean pull (MTCP). For each exercise, 12 participants performed two 1RM sessions tests and two sessions to measure the barbell's load-velocity relationship at 30,45,60,75, and $90 \%$ of 1RM. The velocity recorded at each load was used to establish the linear regression equation and, consequently, to predict 1RM value. A low validity between the 1RM direct test and predicted 1RM was observed for PCK (typical error $[\mathrm{TE}]=3.96$ to $4.50 \mathrm{~kg}$, coefficient of variation $[\mathrm{CV}]=4.68$ to $5.27 \%$, effect size $[\mathrm{ES}]=-0.76$ to -0.58 , BlandAltman bias [BAB] $=9.83$ to $11.19 \mathrm{~kg}$ ), HPK (TE=4.58 to $5.82 \mathrm{~kg}, \mathrm{CV}=6.44$ to $8.14 \%$, $\mathrm{ES}=-0.40$ to -0.39 , $\mathrm{BAB}=3.52$ to $4.17 \mathrm{~kg}$ ), and MTCP $(\mathrm{TE}=6.33$ to $8.08 \mathrm{~kg}, \mathrm{CV}=4.78$ to $6.16 \%, \mathrm{ES}=-0.29$ to $-0.19, \mathrm{BAB}=3.98$ to $6.17 \mathrm{~kg}$ ). Adequate reliability was observed for the 1RM direct test and for the predicted 1RM. However, based on Bland-Altman limits of agreement, lower measurement errors were obtained for the 1RM direct test in comparison to the predicted 1RM for all the exercises. In conclusion, the load-velocity relationship was not able to predict 1RM values with high accuracy in the PCK, HPK, and MTCP. Moreover, the 1RM direct test was the most reliable for PCK, HPK and MTCP.
\end{abstract}

Key words: power clean, maximum strength, velocity-based training, measurement error, linear regression

\section{Introduction}

Weightlifting derivatives (i.e. exercises that omit a portion of the snatch or clean and jerk) allow substantial improvements in muscle power production and sport tasks such as vertical jumps, change of direction running, and sprint performances (Berton, Lixandrao, Pinto, \& Tricoli, 2018; Suchomel, McKeever, \& Comfort, 2020). For this reason, practitioners implement weightlifting derivatives in strength and conditioning programs as an appropriate tool to optimize sports performance (Suchomel, Comfort, \& Stone, 2015).

In order to implement the weightlifting derivatives in strength and power-oriented training program, the procedure to determine adequate loads is a recurrent question that remains as a potential practical barrier. Typically, the training load for weightlifting derivatives with the catch phase (weightlifting catching derivatives) or without catch phase (weightlifting pulling derivatives) is prescribed based on percentages of the one-repetition maximum test (1RM) of a weightlifting catching derivative (Comfort, Jones, \& Udall, 2015; Suchomel \& Sole, 2017). The 1RM test measures the maximum load that an individual can lift for one repetition and is considered the gold standard for assessing the maximal strength during dynamic tasks (Brown \& Weir, 2001; Buckner, et al., 2017). However, the 1RM test has the disadvantage of being very time-consuming, at least in a large group of individuals (e.g. team sports) (Chapman, 
Whitehead, \& Binkert, 1998). Indeed, it has been reported that $1 \mathrm{RM}$ testing for a single exercise can take hours depending on the personnel involved and the number of available stations (Chapman, et al., 1998). Moreover, another disadvantage linked to the $1 \mathrm{RM}$ test is the injury risk (Niewiadomski, et al., 2008). It has been suggested that when performed incorrectly, the 1RM test might expose the athletes to a higher risk of injuries due to the heavy loads lifted (Niewiadomski, et al., 2008). As observed, the $1 \mathrm{RM}$ test has some disadvantages in certain contexts. Consequently, researchers have suggested that applicable practical alternatives might be used to predict the 1RM performance.

There has been great interest in determining 1RM performance based upon the load-velocity relationships (Banyard, Nosaka, \& Haff, 2017; McMaster, Gill, Cronin, \& McGuigan, 2014). In summary, velocity of the bar is measured at different submaximal loads through the loadvelocity continuum and then a linear regression equation may be applied to explain the load-velocity relationships (Banyard, et al., 2017; Garcia-Ramos, et al., 2018a; Loturco, et al., 2017; Loturco, et al., 2016; Ruf, Chery, \& Taylor, 2018). Furthermore, researchers suggested once the regression equation was determined for a given exercise, the 1RM value of that exercise may be estimated with as little as velocity of the first repetition (Loturco, et al., 2017). The use of the load-velocity relationship for predicting the 1RM performance was justified with high validity and reliability values obtained during the bench press and half-squat (Garcia-Ramos, et al., 2018a; Loturco, et al., 2016, 2017; PerezCastilla, Garcia-Ramos, Padial, Morales-Artacho, \& Feriche, 2018). For example, Loturco et al. (2017) reported a high validity of the load-velocity relationship to predict the 1RM performance during the bench press ( $\geq 95 \%$ of accuracy). Loturco et al. (2016) and Perez-Castilla et al. (2018) also reported similar results ( $\geq 94 \%$ of accuracy) during the halfsquat. Regarding reliability, Garcia-Ramos et al. (2018a) have demonstrated the load-velocity relationship for predicting the 1RM performance in the bench press has a low measurement error (intraclass correlation coefficient [ICC] between 0.90 and 0.95 , and coefficient of variation [CV] between $3.05 \%$ and $5.11 \%)$. Similarly, Banyard et al. (2017) reported a low measurement error of the load-velocity relationship for predicting the 1RM performance during the squat $(\mathrm{ICC}=0.92, \mathrm{CV}=5.7 \%$ and standard error of the measurement $[\mathrm{SEM}]=8.6 \mathrm{~kg}$ ).

Given the factors presented previously, it may be suggested the use of the load-velocity relationships to predict the 1RM values in the weightlifting derivatives (Haff, Garcia-Ramos, \& James, 2020; Thompson, Rogerson, Dorrell, Ruddock, \& Barnes, 2020; Thompson, Rogerson, Ruddock, Banyard, \& Barnes, 2021). However, it is important to note that linear regression equations and, consequently, their validity and reliability are specific for each exercise (Perez-Castilla, et al., 2018). Thus, the results found in the bench press and half-squat cannot be extrapolated to the weightlifting derivatives (Perez-Castilla, et al., 2018). Also, studies focused on predicting $1 \mathrm{RM}$ in weightlifting derivatives are scarce (Haff, et al., 2020). Therefore, the purpose of the present study was to investigate the concurrent validity and reliability of the load-velocity relationship to predict the $1 \mathrm{RM}$ value during the weightlifting derivatives power clean from the knee (PCK), high pull from the knee (HPK), and midthigh clean pull (MTCP). It is hypothesized that the load-velocity relationship would present a high degree of validity and reliability for all the exercises.

\section{Methods}

\section{Participants}

Twelve healthy male participants were recruited for this study (age 28.5 \pm 5.2 years, body mass [BM] $80.3 \pm 8.3 \mathrm{~kg}$, body height $1.76 \pm 0.05 \mathrm{~m}$ ). They had at least one year of experience in weightlifting training (average: $3.2 \pm 2.0$ years, range: 1-7 years), and an average of $1 \mathrm{RM}$ to BM ratio in the PCK of $1.1 \pm 0.1 \mathrm{kgkg}^{-1}$ (range: 0.9 to $1.5 \mathrm{kgkg}^{-1}$ ). All participants were informed about the purpose and all the potential risks of the study before signing a consent form. The protocol and consent were approved by the Ethics Committee of the University São Paulo (No. 90499718.7.0000.5391; approval date: July 7, 2018), and they complied with all ethical standards for research involving human participants set by the Declaration of Helsinki.

\section{Procedure}

This study investigated the concurrent validity and reliability of the load-velocity relationship for predicting the 1RM performance of the PCK, HPK, and MTCP. The 1RM direct test of each exercise (PCK, HPK, and MTCP) was selected as the criterion method to assess maximum performances for their respective comparisons. An individualized linear regression equation of the peak vertical barbell-velocity for the five submaximal loads $(30,45,60,75$ and $90 \%$ of $1 \mathrm{RM})$ was developed to predict the 1RM value for the PCK, HPK and MTCP. Thereafter, the predicted peak vertical barbell-velocity at $100 \%$ was used to estimate the $1 \mathrm{RM}$ value. The 1RM direct and 1RM predicted tests were performed on two occasions to determine the between-session reliability of each test, separately. Participants visited the laboratory on 12 occasions; four sessions per exercise. For a given exercise, the first two sessions were dedicated to the 1RM direct tests, while the test to predict the 1RM based on the load-velocity relationship was carried 
out in the last two sessions. A battery of five loads $(30,45,60,75$ and $90 \%$ of 1RM) was employed through the load-velocity continuum to establish a linear regression equation to predict the $100 \%$ of 1RM. Consistent intervals of 72 or 96 hours for each participant were granted between the sessions. The order of the exercises was randomized among all participants to minimize biases.

\section{The 1RM direct test}

The 1RM direct test was assessed for the PCK, HPK and MTCP weightlifting derivatives. For all the exercises, the participants performed a general warm-up on a cycle ergometer at $20 \mathrm{~km} \cdot \mathrm{h}^{-1}$ for five minutes. After a one-minute rest interval, the participants performed a specific warm-up of the same weightlifting exercise that was randomly determined for that day (i.e. either PCK, or HPK, or MTCP). Similar to Suchomel, Wright, Kernozek, and Kline (2014) the specific warm-up consisted of three repetitions at 30,50 , and $70 \%$ followed by one repetition at $90 \%$ of their estimated 1RM. The estimation was determined by the information provided by the participants. Rest intervals of two minutes were provided between the loads. Three minutes after the specific warm-up, the 1RM test was performed. To determine the value of the $1 \mathrm{RM}$, participants had up to five attempts, with a three-minute rest interval between them, following previous guidelines (Brown \& Weir, 2001). The 1RM value was defined as the highest load lifted for each exercise, through previously defined technical parameters (details in Table 1 and Figure 1) (DeWeese, Serrano, Scruggs, \& Burton, 2013; Suchomel, DeWeese, \& Serrano, 2016).

\section{The $1 R M$ predicted test}

For all the exercises, a general and a specific warm-up were performed. The general warm-up was performed on a cycle ergometer at $20 \mathrm{~km} \cdot \mathrm{h}^{-1}$ for five minutes. For the specific warm-up, the participants performed two sets of four repetitions at $45 \%$ of $1 \mathrm{RM}$ of the specific exercise (i.e. either PCK, or HPK, or MTP). Two minutes of rest were allowed between the sets.

Three minutes after the specific warm-up, participants randomly performed one of the three exercises (PCK, HPK or MTCP). The exercises were performed following the guidelines and procedures previously defined (Table 1 and Figure 1). To minimize biases related to the order of loads, all exercises were performed with a battery of loads in a randomized order. The loads analyzed were $30,45,60,75$ and $90 \%$ of $1 \mathrm{RM}$. For loads

Table 1. Technical parameters to assess the 1RM performance of the power clean from the knee (PCK), high pull from the knee (HPK) and mid-thigh clean pull (MTCP)

Acceptable lifts
PCK: In the start position, participants had the barbell supported on the blocks at a
location of just above their knees (i.e. upper edge of the patella), with knees slightly
bent, and hip flexed, shoulders above the bar, back flat, and arms extended with
elbows pointed out (Figure 1B). Participants were asked to initiate the movement,
and at mid-thigh position, to perform an explosive extension of the hips, knees, and
ankles (triple extension) while concomitantly shrugging their shoulders in order to
accelerate the barbell in a vertical motion. Then, participants were asked to catch
the bar in the front rack position (shoulders rotated and flexed ahead with elbows up)
underneath through a downward motion of their center of mass. Finally, participants
were asked to complete the movement through a full extension of the hips and knees
to reach the upright position with the barbell in the front rack position (Figure 1E).

HPK: In the start position, participants followed the same procedures of the PCK (Figure 1C). However, during the HPK participants were asked to perform an explosive triple extension, and then to shrug shoulders straight up while flexing their elbows and pulling the barbell upward at the nipple line. In the final position ( $\max$ barbell displacement) participants were in triple extension. An adjustment procedure for each participant was developed to ensure consistency in the displacement of the barbell between attempts (Figure 1F). Adjustment in displacement was performed with an empty barbell before the general warm-up of the 1RM direct test.

MTCP: In the start position, participants were asked to keep the barbell supported on the blocks at mid-thigh position, with knees and hips slightly flexed, back upright, shoulders above the barbell and arms fully extended (Figure 1D). Then, participants were asked to perform an explosive triple extension in order to accelerate the barbell, while shrugging their shoulders with arms straight, elbows rotated out, and bar close to the body. Participants needed to complete the barbell displacement previously adjusted (i.e. from the position at mid-thigh from the blocks to the max barbell height achieved at the final point of the triple extension with shrugged shoulders). An adjustment procedure for each participant was developed to ensure consistency in the displacement of the barbell between attempts (Figure 1G). Adjustment in displacement was performed with an empty barbell before the general warm-up of the 1RM direct test.

\section{Unacceptable lifts}

The thighs of the participants were below parallel squat position to the floor in the instant of the catch.

The participant was not able to complete the movement to an upright position with the barbell in the front rack (Figure 1E).

The participant did not cover the entire barbell displacement previously adjusted according to his/her anthropometric characteristics.

During the upward phase, the barbell did not contact the elastic bands (Figure 1F) in both sides concomitantly.

The participant did not cover the entire barbell displacement previously adjusted according to his/her anthropometric characteristics.

During the upward phase, the barbell did not contact the elastic bands (Figure 1G) in both sides concomitantly. 

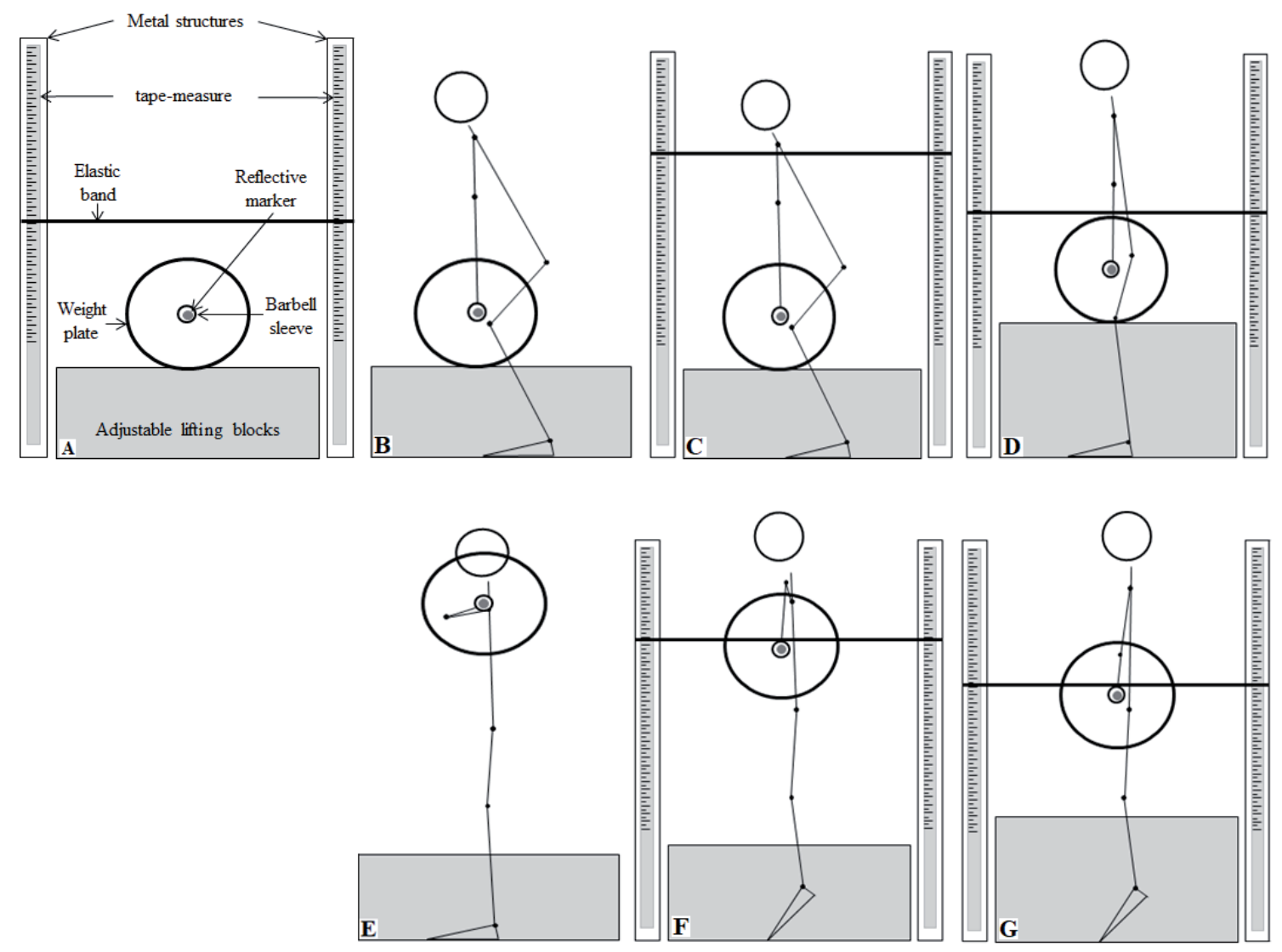

Figure 1. Adjustable metal structures positioned on each side of the barbell (A); starting position of the power clean from the knee $(B)$, high pull from the knee $(C)$, and mid-thigh clean pull (D). Final position of the power clean from the knee (E), high pull from the knee $(F)$, and mid-thigh clean pull $(G)$.

of $30,45,60$, and $75 \% 1 \mathrm{RM}$ sets of three repetitions were performed, while at $90 \% 1 \mathrm{RM}$ only two repetitions were performed. The participants were instructed to perform all repetitions as fast as possible (Banyard, et al., 2017; Garcia-Ramos, et al., 2018a). A 30-second rest interval was allowed between the repetitions. Moreover, two-minute rest intervals were granted between the sets (Suchomel, et al., 2014).

\section{Data collection}

Video recordings of all the repetitions were obtained via an iPhone $5 \mathrm{~s}$ camera (Apple Inc., USA) and were filmed with 1,280 x 720 pixel resolution at 120 frames per second (FPS) (Garhammer \& Newton, 2013). Throughout all video recordings, the iPhone's camera was placed on a tripod 1.30 $\mathrm{m}$ above the ground, in the sagittal plane (participants' left side) at $5 \mathrm{~m}$ from the area in which the exercises were performed. A black background and a reflective marker placed on the left side of the Olympic barbell were used to allow better contrast and, consequently, accuracy in the auto-tracking of the barbell trajectory.

Barbell trajectory auto-tracking analyses were carried out by the Kinovea software (Experimental Version 0.8.25-x64) (Daehlin, Krosshaug, \& Chiu,
2017; Garhammer \& Newton, 2013). Kinovea is an appropriate option as it is easy-to-use, requires no experience to obtain accurate and reliable measurements, is a portable and free tool (i.e. can be used in real field context and have no cost), and has been used to assesses different sporting tasks (BalsalobreFernandez, Tejero-Gonzalez, del Campo-Vecino, \& Bavaresco, 2014; Grigg, Haakonssen, Rathbone, Orr, \& Keogh, 2018; Pueo, Penichet-Tomas, \& Jimenez-Olmedo, 2020; Puig-Divi, et al., 2019). For the correct measurement, each video analysis was calibrated with the same reference length (weight plate of $45 \mathrm{~cm}$ diameter) and by the same investigator (Garhammer \& Newton, 2013). After this step, an auto-tracking procedure was performed for all the repetitions. For all the exercises, the movement start was defined when the weight plates were resting on the blocks. It should be noted that for each participant, the height of the blocks was individually adjusted prior to the beginning of the testing session. The barbell was supported on the blocks to ensure the same vertical barbell displacement for all the repetitions and across the loads. However, the end of the movement differed between exercises. For the PCK, the end of the movement was defined as the first negative peak velocity that occurred after the start of the lift (Balsalobre-Fernandez, Geiser, 
Krzyszkowski, \& Kipp, 2020), whereas for the HPK and MTCP the end was defined as the frame that barbell touched the elastic band (Figure 1). Finally, peak vertical barbell velocity was obtained from vertical axis (y-axis) in *xlsx files for subsequent statistical analysis. Only the repetition with the highest peak vertical barbell velocity at each load was considered for statistical analysis. It is important to highlight that for concentric-only exercises the use of peak velocity is considered appropriate as it presents a similar result when compared to the mean velocity, or the mean propulsive velocity (Garcia-Ramos, Pestaña-Melero, Pérez-Castilla, Rojas, \& Haff, 2018b).

\section{Statistical analyses}

All data are presented as mean $\pm \mathrm{SD}$, where appropriate. Statistical analyses were carried out for each exercise separately. The first procedure applied was the Shapiro-Wilk's test to verify normal distribution. Then, the peak vertical barbell velocity was estimated at $1 \mathrm{RM}(100 \%)$ for each participant. We then performed an individualized linear regression equation (Thompson, et al., 2021) between the peak vertical barbell velocity and each of the five submaximal loads (30, 45, 60, 75 and $90 \%$ of 1RM). Thereafter, a second linear regression was carried out to predict the 1RM estimate. The peak vertical barbell velocity value at $100 \% 1 \mathrm{RM}$ was used in the linear regression equation to predict the 1RM value. In addition, the linear regression equation to predict the $1 \mathrm{RM}$ value was assessed by the coefficient of determination $\left(\mathrm{R}^{2}\right)$ and $\mathrm{p}$-value. The significance level was set at $\mathrm{p} \leq .05$.

To determine validity, the best performance of the two 1RM direct tests was compared to the predicted 1RM test of sessions 1 and 2. Validity was assessed with typical error (TE), coefficient of variation (CV) (Hopkins, 2000), Hedges' g effect size (ES) and the associated $95 \%$ confidence intervals (CI) (Nakagawa \& Cuthill, 2007). ESs were interpreted as: trivial $(0$ to $<0.2)$, small $(0.2$ to $<0.6)$, moderate $(0.6$ to $<1.1)$ and large $(1.2$ to 2.0$)$ based on previous guidelines (Hopkins, Marshall, Batterham, \& Hanin, 2009). However, if the CI overlapped thresholds for substantial positive and negative values, the effect was considered unclear (Nakagawa \& Cuthill, 2007). In addition, BlandAltman plots (limits of agreement at $95 \%$ and bias) were used to evaluate the agreement of the 1RM value between the 1RM direct test (best performance) and the two predicted tests (session 1 and session 2) (Bland \& Altman, 1986).

Between-sessions reliability was evaluated for the 1RM direct and predicted tests separately. For that, the two-way mixed method intraclass correlation coefficient (ICC) and associated 95\% CI were calculated. The ICC was interpreted based on the recommendations of Koo and $\mathrm{Li}$ (2016), where less than 0.50 , between 0.50 and 0.75 , between 0.75 and 0.90 , and greater than 0.90 indicated as poor, moderate, good, and excellent reliability, respectively. In addition, TE and CV (Hopkins, 2000), ES and the associated 95\% CI (Nakagawa \& Cuthill, 2007), and Bland-Altman plots (limits of agreement at $95 \%$ and bias) were also utilized (Bland \& Altman, 1986).

Finally, a post-hoc power analysis using $\mathrm{G}^{*}$ Power version 3.1.9.7 software was performed to determine the present study's statistical power. The post-hoc analysis was calculated for each linear equation that predicted 1RM value for PCK, HPK, and MTCP. The input parameters were the $\mathrm{R}^{2}$ of each linear equation, $\mathrm{ES} \mathrm{f}^{2}$, an alpha level of 0.05 , total sample size, and the number of predictors.

\section{Results}

The Shapiro-Wilk's test of normality revealed all data were normally distributed ( $p>05)$. The estimated peak vertical barbell velocity at $100 \%$ for the PCK was $1.93 \pm 0.12 \mathrm{~m} \cdot \mathrm{s}^{-1}$ for session 1 and $1.84 \pm 0.16 \mathrm{~m} \cdot \mathrm{s}^{-1}$ for session 2 . For the HPK, the estimated peak vertical barbell velocities at $100 \%$ were $2.08 \pm 0.19 \mathrm{~m} \cdot \mathrm{s}^{-1}$ and $2.17 \pm 0.22 \mathrm{~m} \cdot \mathrm{s}^{-1}$ for sessions 1 and 2, respectively. Finally, during the MTCP the estimated peak vertical barbell velocities at $100 \%$ were $0.74 \pm 0.20 \mathrm{~m} \cdot \mathrm{s}^{-1}$ and $0.70 \pm 0.13 \mathrm{~m} \cdot \mathrm{s}^{-1}$ for sessions 1 and 2, respectively. A second linear regression equation was carried out between all peak vertical barbell velocities and their respective loads. The $\mathrm{R}^{2}$ were $0.60(\mathrm{p}<.001)$ and $0.66(\mathrm{p}<.001)$ for the PCK, $0.87(\mathrm{p}<.001)$ and $0.82(\mathrm{p}<.001)$ for the HPK, and $0.84(\mathrm{p}<.001)$ and $0.91(\mathrm{p}<.001)$ for the MTCP for sessions 1 and 2, respectively (Figure 2).

For validity, the load-velocity relationship underestimated $1 \mathrm{RM}$ values when compared with the 1RM direct test. For the PCK, the best 1RM direct test was $90.42 \pm 16.34 \mathrm{~kg}$, whereas lower values were observed for the estimated $1 \mathrm{RM}$ test of sessions $1(78.51 \pm 14.83 \mathrm{~kg})$ and $2(80.57 \pm 17.39)$. For the HPK, the best 1RM direct test was $73.27 \pm 9.56$ $\mathrm{kg}$, whereas lower values were observed for the estimated 1RM test of sessions $1(69.74 \pm 7.94 \mathrm{~kg})$ and $2(69.10 \pm 11.73)$. Finally, the best $1 \mathrm{RM}$ direct test in the MTCP was $134.40 \pm 21.32 \mathrm{~kg}$, whereas lower values were observed for the estimated $1 \mathrm{RM}$ test of sessions 1 and $2(127.68 \pm 24.13$ and $130.41 \pm 20.86$, respectively). The results of the validity analysis are presented in Table 2 (TE, CV, and ES) and Figure 3 (limits of agreement at $95 \%$, and bias).

For reliability, the results are presented in Table 3 and Figure 4. For the PCK, HPK, and MTCP higher reliability was found for the 1RM direct test compared to the estimated 1RM test. 

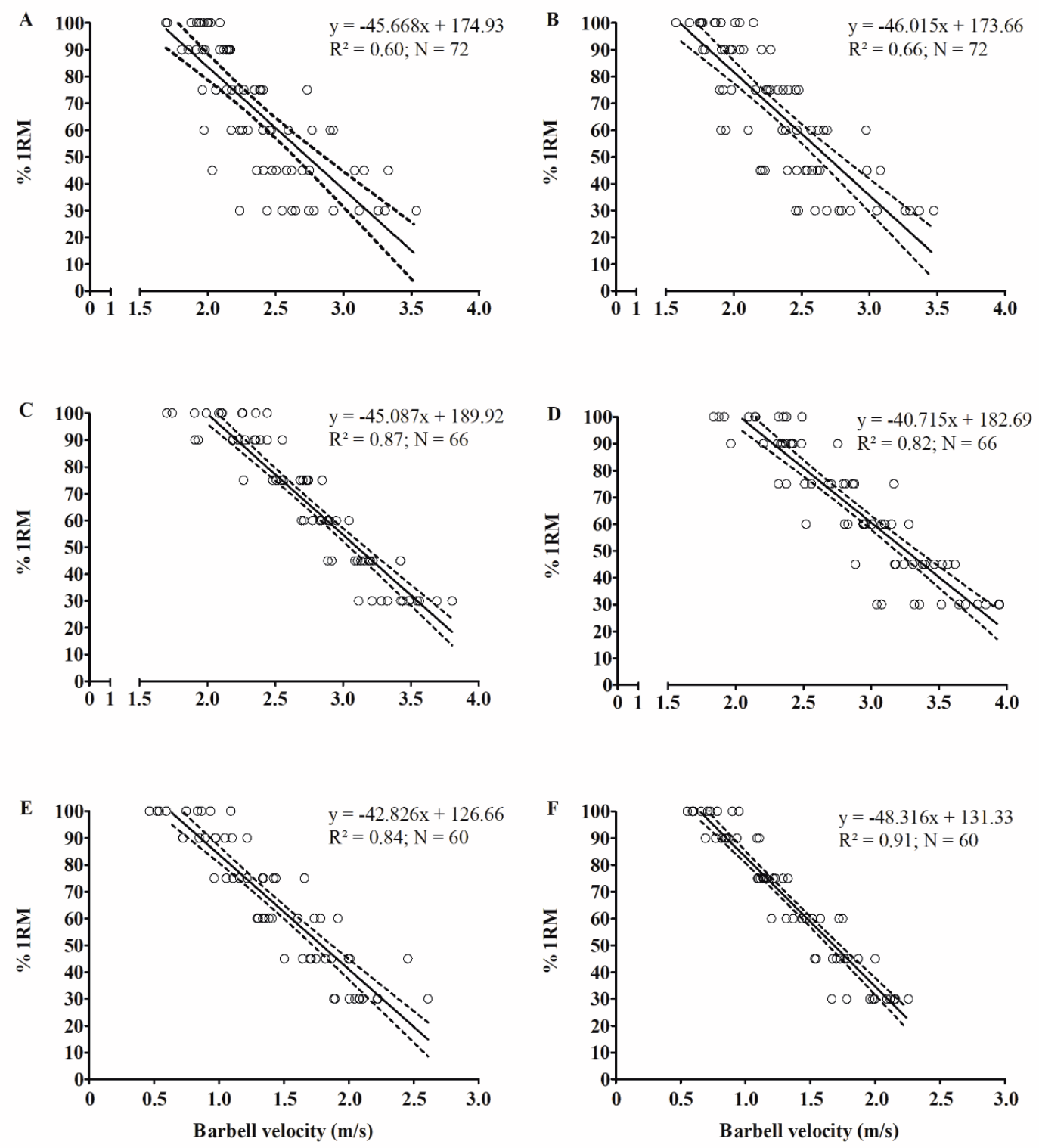

Figure 2. Relationship between barbell-velocity and percentages of $1 R M$ (loads 30, 45, 60, 75, 90 and 100\% 1RM). (A) PCK session 1, (B) PCK session 2, (C) HPK session 1, (D) HPK session 2, (E) MTCP session 1, and (F) MTCP session 2. Dotted lines represent confidence interval ( $95 \%)$. $R 2=$ coefficient of determination; $N=$ number of raw data included in the regression analysis.

Table 2. Validity between the best 1RM direct test vs. predicted 1RM-session 1, and best 1RM direct test vs. predicted 1RMsession 2 of the exercises power clean from the knee (PCK), high pull from the knee (HPK) and mid-thigh clean pull (MTCP)

\begin{tabular}{lcc}
\hline & $\begin{array}{c}\text { Best 1RM direct test vs. } \\
\text { predicted 1RM - Session 1 }\end{array}$ & $\begin{array}{c}\text { Best 1RM direct test vs. } \\
\text { predicted 1RM - Session 2 }\end{array}$ \\
\hline PCK & $3.96(2.81,6.72)$ & \\
TE $(\mathrm{kg})$ & $4.68(3.30,7.90)$ & $5.50(3.19,7.66)$ \\
CV $(\%)$ & $-0.76(-1.27,-0.31)$ & $-0.58(-1.06,-0.15)$ \\
ES & & \\
HPK & $5.82(4.07,10.22)$ & $4.58(3.21,8.05)$ \\
TE $(\mathrm{kg})$ & $8.14(5.80,10.00)$ & $6.44(4.60,11.00)$ \\
CV $(\%)$ & $-0.40(-0.53,0.35)$ & $-0.39(-0.53,0.34)$ \\
ES & & \\
MTCP & $8.08(5.56,14.76)$ & $6.33(4.36,11.57)$ \\
TE $(\mathrm{kg})$ & $6.16(4.30,11.00)$ & $4.78(3.30,8.40)$ \\
CV $(\%)$ & $-0.29(-0.53,0.39)$ & $-0.19(-0.51,0.41)$ \\
ES &
\end{tabular}

Note. TE=typical error, $C V=$ coefficient of variation, ES=Hedges' $g$ effect size. Data are presented as mean and confidence interval $95 \%$. 

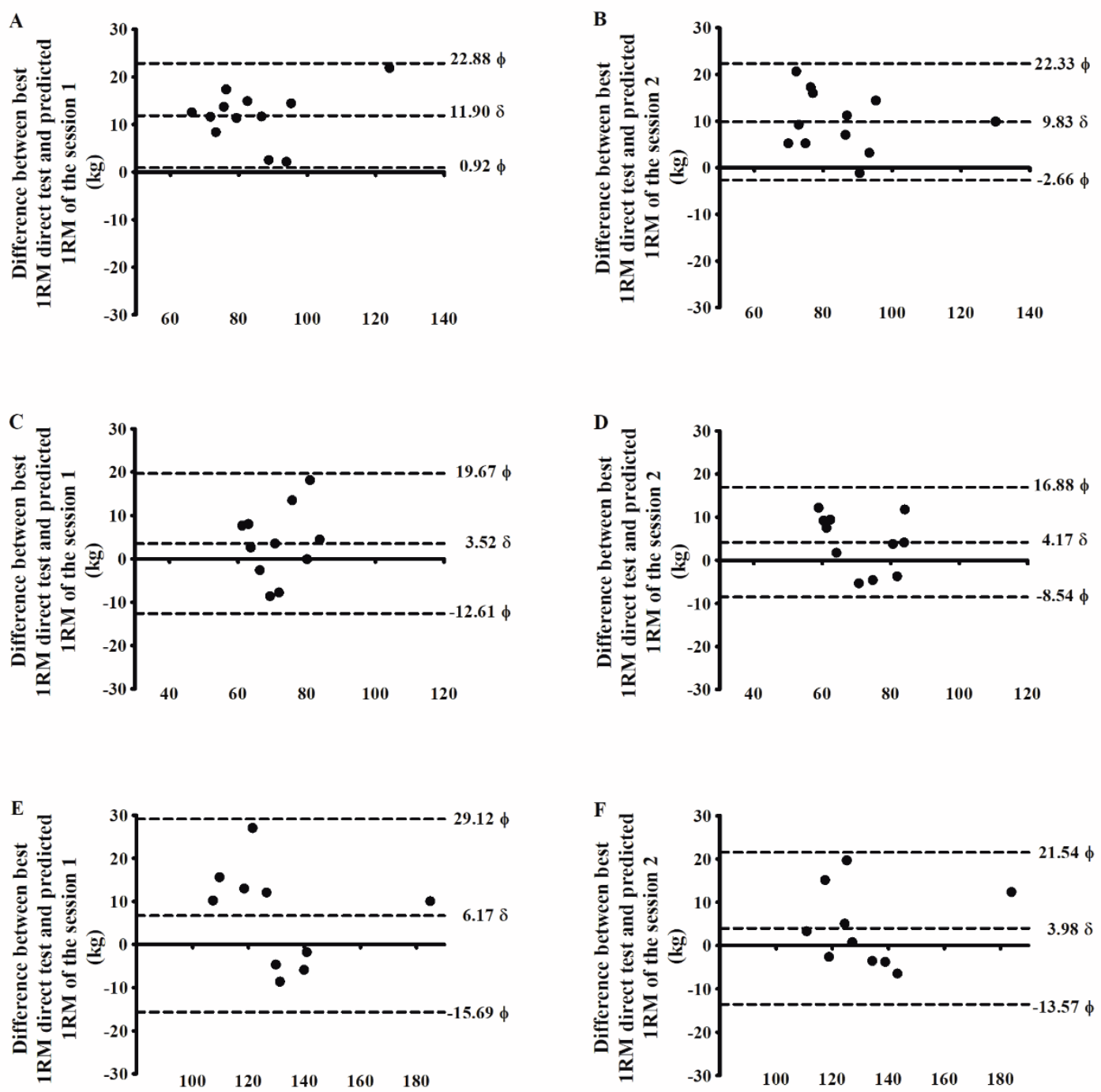

Mean between best 1RM

direct test and predicted $1 \mathrm{RM}$ of the session $1(\mathrm{~kg})$

Mean between best 1RM direct test and predicted 1RM of the session $2(\mathrm{~kg})$

Figure 3. Validity analysis. Bland-Altman plots showing differences between (A) best 1RM direct test vs. predicted 1RM-session 1 , and (B) best 1RM direct test vs. predicted 1RM-session 2 for PCK; (C) best 1RM direct test vs. predicted 1RM-session 1, and (D) best 1RM direct test vs. predicted 1RM - session 2 for HPK; (E) best 1RM direct test vs. predicted 1RM-session 1, and (F) best $1 R M$ direct test $v$ s. predicted $1 R M-$ session 2 for MTCP. $\delta=$ bias; $\theta=$ superior and inferior limits of agreement.

Table 3. Reliability between IRM direct test and predicted 1RM based on the load-velocity relationship

\begin{tabular}{lcccccc}
\hline Tests & Session 1 $(\mathrm{kg})$ & Session 2 $(\mathrm{kg})$ & ICC & TE $(\mathrm{kg})$ & CV $(\%)$ & ES \\
PCK & & & & & & \\
1RM direct test & $89.33 \pm 16.62$ & $88.91 \pm 16.85$ & $0.97(0.89-0.99)$ & $3.04(2.15-5.16)$ & $3.41(2.40-5.80)$ & $-0.02(-0.44-0.39)$ \\
Predicted 1RM & $78.51 \pm 14.83$ & $80.57 \pm 17.39$ & $0.97(0.83-0.99)$ & $3.84(2.72-6.52)$ & $4.82(3.40-8.20)$ & $0.13(-0.29-0.55)$ \\
HPK & & & & & & \\
1RM direct test & $71.18 \pm 8.22$ & $72.68 \pm 9.68$ & $0.91(0.64-0.98)$ & $2.58(1.81-4.54)$ & $3.59(2.50-6.30)$ & $0.17(-0.27-0.61)$ \\
Predicted 1RM & $69.74 \pm 7.94$ & $69.10 \pm 11.73$ & $0.73(0.25-0.93)$ & $5.26(3.68-9.24)$ & $7.58(5.40-13.00)$ & $-0.06(-0.51-0.37)$ \\
MTCP & & & & & & \\
1RM direct test & $133.00 \pm 21.78$ & $133.90 \pm 21.55$ & $0.98(0.95-0.99)$ & $1.86(1.28-3.40)$ & $1.39(0.97-2.40)$ & $0.04(-0.40-0.48)$ \\
Predicted 1RM & $127.68 \pm 24.13$ & $130.41 \pm 20.86$ & $0.95(0.73-0.99)$ & $6.90(4.75-12.60)$ & $5.34(3.70-9.40)$ & $0.12(-0.34-0.59)$ \\
\hline
\end{tabular}

Note. PCK=power clean from the knee, HPK=high pull from the knee, MTCP=mid-thigh clean pull.

$I C C=$ intraclass correlation coefficient, TE=typical error, $C V=$ coefficient of variation, and $E S=$ Hedges' $g$ effect size (data are presented as mean and confidence interval 95\%). Sessions 1 and 2=data are presented as mean and standard deviation. 

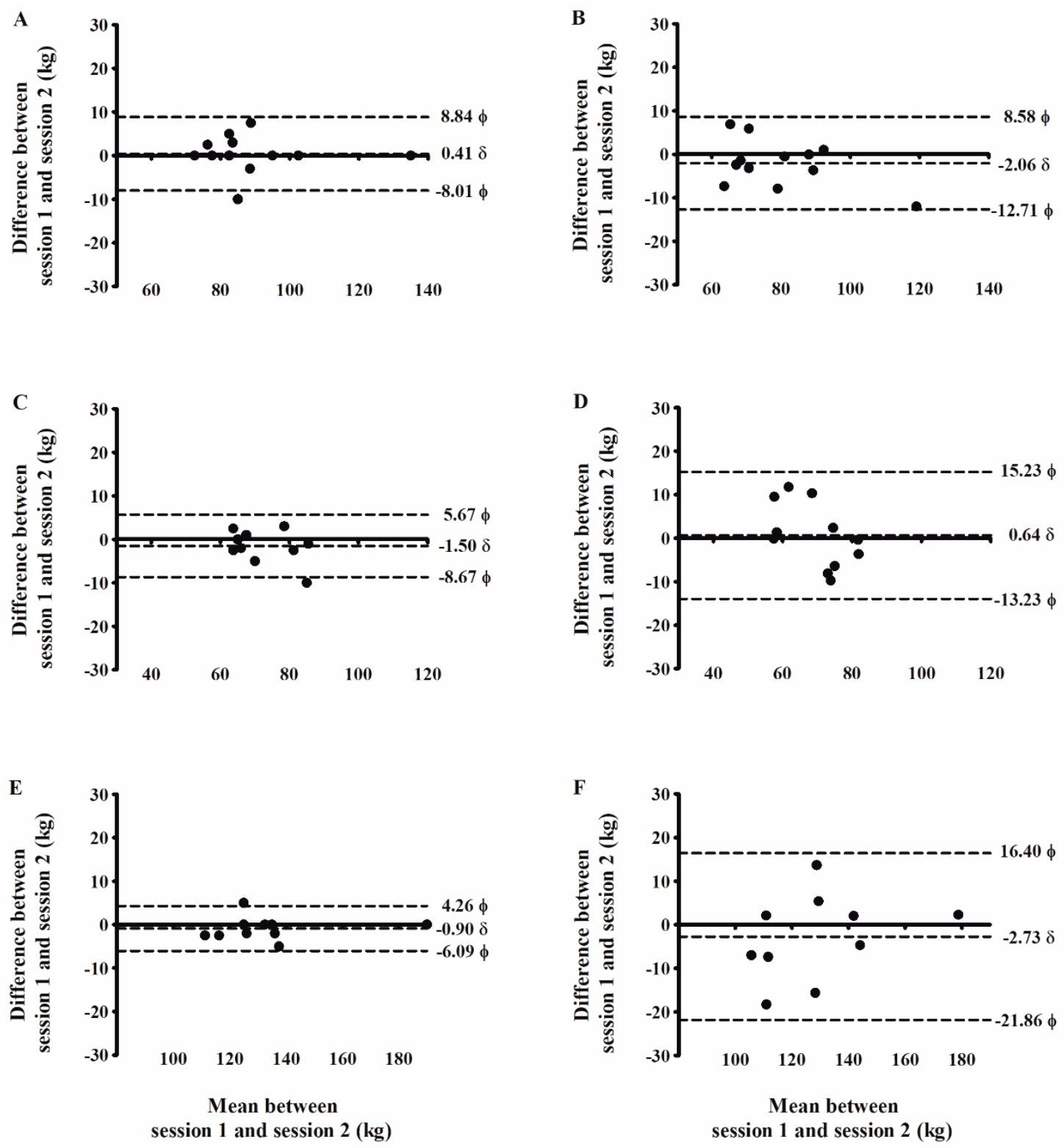

Figure 4. Reliability analysis. Bland-Altman plots showing differences between (A) 1RM direct test - sessions 1 and 2, and (B) predicted 1RM - sessions 1 and 2 for PCK; (C) IRM direct test - sessions 1 and 2, and (D) predicted $1 R M$ - sessions 1 and 2 for HPK; (E) 1RM direct test - sessions 1 and 2, and (F) predicted $1 R M-$ sessions 1 and 2 for MTCP. $\delta=$ bias, $\theta=$ superior and inferior limits of agreement.

\section{Discussion and conclusions}

The purpose of this study was to investigate the concurrent validity and reliability of the loadvelocity relationship to predict $1 \mathrm{RM}$ values in the PCK, HPK and MTCP. It was hypothesized that for all the exercises the load-velocity relationships would present high validity and reliability. However, the main finding of this study is that the load-velocity relationship is not a suitable method to predict the 1RM performance accurately during the PCK, HPK and MTCP. In addition, although the 1RM predictions based on the load-velocity relationship demonstrated high reliability for the three exercises, better reliability and lower measurement errors were reported for the 1RM direct test during the three exercises.
In the present study, the linearity of the loadvelocity relationship was lower for PCK in both $1 \mathrm{RM}$ prediction sessions $\left(\mathrm{R}^{2}=0.60\right.$ and 0.66$)$, compared to HPK $\left(\mathrm{R}^{2}=0.82\right.$ and 0.87$)$ and MTCP $\left(\mathrm{R}^{2}=0.84\right.$ and 0.91) (Figure 2). Although greater linearity of the load-velocity relationship was observed for the HPK and MTCP, the results of the three exercises did not corroborate previous findings in the literature. Strong linearity $\left(\mathrm{R}^{2} \geq 0.94\right)$ in the load-velocity relationship was reported for the bench press and half-squat exercises in previous research (Loturco, et al., 2016, 2017; Perez-Castilla, et al., 2018). These differences may be attributable to technical parameters, in which the load-velocity relationship has proven to be task-specific (PerezCastilla, et al., 2018). Nonetheless, the explanation 
for the different results between our study and the literature is not entirely clear and more research is warranted.

Strong linearity for the load-velocity relationship is necessary for an accurate prediction of the $1 \mathrm{RM}$ value (Garcia-Ramos, et al., 2018a). As aforementioned, our results did not demonstrate strong linearity based on $\mathrm{R}^{2}$ values ( 0.60 to 0.91 ) (Figure 2). In this sense, a poor validity of the load-velocity relationship was observed (Table 2 and Figure 3). In this study, the predicted 1RM values were underestimated in sessions 1 and 2 when compared to the $1 \mathrm{RM}$ direct tests of the three weightlifting derivatives. Researchers reported results support the low accuracy of the load-velocity relationship to predict the $1 \mathrm{RM}$ value in some exercises. For instance, Ruf et al. (2018) showed that the load-velocity relationship overestimated the estimated 1RM value compared to the 1RM direct test for the deadlift. Similarly, Banyard et al. (2017) reported large absolute errors (10.6 to $17.2 \mathrm{~kg}$ ) and poor CVs (7.4 to $12.8 \%$ ) between the estimated 1RM value and the $1 \mathrm{RM}$ direct test for the back squat. In contrast, Loturco et al. $(2016,2017)$ demonstrated low CV between the estimated 1RM value and the 1RM direct test for the half-squat $(0.30$ to $0.75 \%)$ and bench press exercises (1.12 to $1.15 \%$ ), respectively. In addition, Garcia-Ramos et al. (2018a) demonstrated low systematic bias between the predicted $1 \mathrm{RM}$ and the $1 \mathrm{RM}$ actual test $(-2.3$ to $0.5 \mathrm{~kg})$ for the bench press exercise. Therefore, the load-velocity relationship seems to be an adequate option for predicting the 1RM value. As noted, the accuracy of the load-velocity relationship to predict the 1RM value may vary based on the exercise. For the three weightlifting derivatives (PCK, HPK, and MTCP) evaluated in this study, the use of the load-velocity relationship to predict the 1RM value is not recommended.

The analyses showed an acceptable reliability and low validity of the predicted $1 \mathrm{RM}$ values for the three weightlifting derivatives. However, whether the reliability of the $1 \mathrm{RM}$ value predicted by the load-velocity relationship was considered adequate, better results were found for the 1RM direct test (Table 3 and Figure 4). These results are in line with previous research (Banyard, et al., 2017; Garcia-Ramos, et al., 2018a). For example, Banyard et al. (2017) reported small absolute errors $(2.9 \mathrm{~kg})$ and good CVs (2.1\%) for the 1RM direct test during the back squat exercise, while moderate to large absolute errors (8.6 to $16.8 \mathrm{~kg}$ ) and poor CVs (5.7 to $12.2 \%$ ) for the estimated $1 \mathrm{RM}$ value. Similarly, Garcia-Ramos et al. (2018a) showed better relia- bility for the bench press $1 \mathrm{RM}$ direct test $(\mathrm{CV}=$ $1.97 \%$ ) compared to the predicted $1 \mathrm{RM}$ value (CV $=3.8$ to $5.1 \%$ ). In this study, the higher reliability of the 1RM direct test may be evidenced through the difference in the width of limits of agreement of the Bland-Altman plots (Figure 4). For example, the PCK limits of agreement for the 1RM direct test were between -8.01 to $8.89 \mathrm{~kg}$, whereas an interval slightly higher was found for the predicted 1RM value $(-12.7$ to $8.58 \mathrm{~kg})$. In contrast, substantial differences were observed in the limits of agreement for the HPK and MTCP. HPK limits of agreement were observed between -8.67 to $5.67 \mathrm{~kg}$ for the $1 \mathrm{RM}$ direct test and -13.23 to $15.23 \mathrm{~kg}$ for the predicted 1RM value. Similarly, MTCP limits of agreement were observed between -6.09 to $4.26 \mathrm{~kg}$ for the 1RM direct test, and -21.86 to $16.40 \mathrm{~kg}$ for the $1 \mathrm{RM}$ predicted value. In addition, only in the HPK was observed a higher ICC for the 1RM direct test $(\mathrm{ICC}=0.91)$ compared to the estimated $1 \mathrm{RM}$ value (ICC $=0.73$ ) (Table 3). Similarly, only for the MTCP lower TE and CV values were observed for the 1RM direct test compared to the estimated 1RM value (Table 3). Taken together, the 1RM direct test seems to be more reliable than the predicted 1RM values based on the load-velocity relationship.

Three limitations in the present study need to be acknowledged. First, in this study a kinematic approach using a high-speed camera (i.e. $\geq 240$ frames per second) and three-dimensional motion capture (i.e. gold standard) was not performed (Lorenzetti, Lamparter, \& Luthy, 2017; Pueo, 2016). Although it may be a limitation, from a practical standpoint, few coaches have access to this equipment. In this sense, the data collected by a smartphone and the use of a free software, may favor greater implementation and usability. Second, as noted only two sessions to predict the 1RM value was performed. Perhaps more testing sessions could improve the accuracy to predict the 1RM value. However, multiple testing sessions are impractical especially in professional teams with large rosters. Third, sample size of the present study was relatively small, and thus the generalization of the results should be performed with caution. Nevertheless, even with a small sample size, the post-hoc power analysis results demonstrated an adequate power $(\geq 0.85)$ for all the exercises.

In conclusion, the load-velocity relationship showed low validity to predict the 1RM value for PCK, HPK and MTCP. Furthermore, these results have also demonstrated that although a high reliability of the predicted $1 \mathrm{RM}$ value may be expected, the 1RM direct test should be the preferred option. 


\section{References}

Balsalobre-Fernandez, C., Geiser, G., Krzyszkowski, J., \& Kipp, K. (2020). Validity and reliability of a computervision-based smartphone app for measuring barbell trajectory during the snatch. Journal of Sports Sciences, 38(6), 710-716.

Balsalobre-Fernandez, C., Tejero-Gonzalez, C.M., del Campo-Vecino, J., \& Bavaresco, N. (2014). The concurrent validity and reliability of a low-cost, high-speed camera-based method for measuring the flight time of vertical jumps. Journal of Strength and Conditioning Research, 28(2), 528-533.

Banyard, H.G., Nosaka, K., \& Haff, G.G. (2017). Reliability and validity of the load-velocity relationship to predict the 1RM back squat. Journal of Strength and Conditioning Research, 31(7), 1897-1904.

Berton, R., Lixandrao, M.E., Pinto, E.S.C.M., \& Tricoli, V. (2018). Effects of weightlifting exercise, traditional resistance and plyometric training on countermovement jump performance: A meta-analysis. Journal of Sports Sciences, 36(18), 2038-2044.

Bland, J.M., \& Altman, D.G. (1986). Statistical methods for assessing agreement between two methods of clinical measurement. Lancet, 8(1), 307-310.

Brown, L.E., \& Weir, J.P. (2001). Procedures recommendation I: Accurate assessment of muscular strength and power. Journal of Exercise Physiology, 4(3), 1-21.

Buckner, S.L., Jessee, M.B., Mattocks, K.T., Mouser, J.G., Counts, B.R., Dankel, S.J., \& Loenneke, J.P. (2017). Determining strength: A case for multiple methods of measurement. Sports Medicine, 47(2), 193-195.

Chapman, P.P., Whitehead, J.R., \& Binkert, R.H. (1998). The 225-lb reps-to-fatigue test as a submaximal estimate of 1-RM bench press performance in college football players. Journal of Strength and Conditioning Research, 12(4), 258-261.

Comfort, P., Jones, P.A., \& Udall, R. (2015). The effect of load and sex on kinematic and kinetic variables during the mid-thigh clean pull. Sports Biomechanics, 14(2), 139-156.

Daehlin, T.E., Krosshaug, T., \& Chiu, L.Z.F. (2017). Enhancing digital video analysis of bar kinematics in weightlifting: A case study. Journal of Strength and Conditioning Research, 31(6), 1592-1600.

DeWeese, B.H., Serrano, A.J., Scruggs, S.K., \& Burton, J.D. (2013). The midthigh pull: Proper application and progressions of a weightlifting movement derivative. Strength and Conditioning Journal, 35(6), 1-5.

Garcia-Ramos, A., Haff, G.G., Pestana-Melero, F.L., Perez-Castilla, A., Rojas, F.J., Balsalobre-Fernandez, C., \& Jaric, S. (2018a). Feasibility of the 2-point method for determining the 1-repetition maximum in the bench press exercise. International Journal of Sports Physiology and Performance, 13(4), 474-481.

Garcia-Ramos, A., Pestaña-Melero, F.L., Pérez-Castilla, A., Rojas, F.J., \& Haff, G.G. (2018b). Mean velocity vs. mean propulsive velocity vs. peak velocity: Which variable determines bench press relative load with higher reliability? Journal of Strength and Conditioning Research, 32(5), 1273-1279.

Garhammer, J., \& Newton, H. (2013). Applied video analysis for coaches: Weightlifting examples. International Journal of Sports Science and Coaching, 8(3), 581-593.

Grigg, J., Haakonssen, E., Rathbone, E., Orr, R., \& Keogh, J.W.L. (2018). The validity and intra-tester reliability of markerless motion capture to analyse kinematics of the BMX Supercross gate start. Sports Biomechanics, 17(3), 383-401.

Haff, G.G., Garcia-Ramos, A., \& James, L.P. (2020). Using velocity to predict the maximum dynamic strength in the power clean. Sports (Basel), 8(9), 129.

Hopkins, W.G. (2000). Measures of reliability in sports medicine and science. Sports Medicine, 30(1), 1-15.

Hopkins, W.G., Marshall, S.W., Batterham, A.M., \& Hanin, J. (2009). Progressive statistics for studies in sports medicine and exercise science. Medicine and Science in Sports and Exercise, 41(1), 3-13.

Koo, T.K., \& Li, M.Y. (2016). A guideline of selecting and reporting intraclass correlation coefficients for reliability research. Journal of Chiropractic Medicine, 15(2), 155-163.

Lorenzetti, S., Lamparter, T., \& Luthy, F. (2017). Validity and reliability of simple measurement device to assess the velocity of the barbell during squats. BMC Research Notes, 10(1), 707.

Loturco, I., Kobal, R., Moraes, J.E., Kitamura, K., Cal Abad, C.C., Pereira, L.A., \& Nakamura, F.Y. (2017). Predicting the maximum dynamic strength in bench press: The high precision of the bar velocity approach. Journal of Strength and Conditioning Research, 31(4), 1127-1131.

Loturco, I., Pereira, L.A., Cal Abad, C.C., Gil, S., Kitamura, K., Kobal, R., \& Nakamura, F.Y. (2016). Using bar-velocity to predict the maximum dynamic strength in the half-squat exercise. International Journal of Sports Physiology and Performance, 11(5), 697-700.

McMaster, D.T., Gill, N., Cronin, J., \& McGuigan, M. (2014). A brief review of strength and ballistic assessment methodologies in sport. Sports Medicine, 44(5), 603-623.

Nakagawa, S., \& Cuthill, I.C. (2007). Effect size, confidence interval and statistical significance: A practical guide for biologists. Biological Reviews of the Cambridge Philosophical Society, 82(4), 591-605.

Niewiadomski, W., Laskowska, D., Gąsiorowska, A., Cybulski, G., Strasz, A., \& Langfort, J. (2008). Determination and prediction of one repetition maximum (1RM): Safety considerations. Journal of Human Kinetics, 19(1), 109-120. 
Perez-Castilla, A., Garcia-Ramos, A., Padial, P., Morales-Artacho, A.J., \& Feriche, B. (2018). Load-velocity relationship in variations of the half-squat exercise: Influence of execution technique. Journal of Strength and Conditioning Research, 34(4), 1024-1031.

Pueo, B. (2016). High speed cameras for motion analysis in sports science. Journal of Human Sport and Exercise, $1(11), 53-73$.

Pueo, B., Penichet-Tomas, A., \& Jimenez-Olmedo, J.M. (2020). Validity, reliability and usefulness of smartphone and kinovea motion analysis software for direct measurement of vertical jump height. Physiology and Behavior, 227(1), 113144.

Puig-Divi, A., Escalona-Marfil, C., Padulles-Riu, J.M., Busquets, A., Padulles-Chando, X., \& Marcos-Ruiz, D. (2019). Validity and reliability of the Kinovea program in obtaining angles and distances using coordinates in 4 perspectives. PLoS One, 14(6), e0216448.

Ruf, L., Chery, C., \& Taylor, K.L. (2018). Validity and reliability of the load-velocity relationship to predict the one-repetition maximum in deadlift. Journal of Strength and Conditioning Research, 32(3), 681-689.

Suchomel, T.J., Comfort, P., \& Stone, M.H. (2015). Weightlifting pulling derivatives: Rationale for implementation and application. Sports Medicine, 45(6), 823-839.

Suchomel, T.J., DeWeese, B.H., \& Serrano, A.J. (2016). The power clean and power snatch from the knee. Strength and Conditioning Journal, 38(4), 98-105.

Suchomel, T.J., McKeever, S.M., \& Comfort, P. (2020). Training with weightlifting derivatives: The effects of force and velocity overload stimuli. Journal of Strength and Conditioning Research, 34(7), 1808-1818.

Suchomel, T.J., \& Sole, C.J. (2017). Power-time curve comparison between weightlifting derivatives. Journal of Sports Science and Medicine, 16(3), 407-413.

Suchomel, T.J., Wright, G.A., Kernozek, T.W., \& Kline, D.E. (2014). Kinetic comparison of the power development between power clean variations. Journal of Strength and Conditioning Research, 28(2), 350-360.

Thompson, S.W., Rogerson, D., Dorrell, H.F., Ruddock, A., \& Barnes, A. (2020). The reliability and validity of current technologies for measuring barbell velocity in the free-weight back squat and power clean. Sports (Basel), 8(7), 94.

Thompson, S.W., Rogerson, D., Ruddock, A., Banyard, H.G., \& Barnes, A. (2021). Pooled versus individualized loadvelocity profiling in the free-weight back squat and power clean. International Journal of Sports Physiology and Performance, 16(6), 825-833.

Submitted: February 23, 2021

Accepted: July 5, 2021

Published Online First: November 12, 2021

Correspondence to:

Ricardo Berton, Ph.D.

School of Physical Education and Sport, University of São Paulo, São Paulo, Brazil

Av. Prof. Mello Moraes, 65, Butantã, 05508-030, São

Paulo, SP, Brazil

Phone: +55 1130912139

E-mail: ricardoberton88@gmail.com 